\title{
A national survey of COVID-19 challenges, responses and effects in Australian general practice
}

\author{
Rebecca Kippen, Belinda O'Sullivan, \\ Helen Hickson, Michael Leach, \\ Glen Wallace
}

\begin{abstract}
Background and objective Characterising the general practice response to the COVID-19 pandemic is important for ongoing policy planning. The objective of this study was to explore challenges, responses and effects of COVID-19 in Australian general practice in the early stages of the pandemic, and to consider variance by geographic location.
\end{abstract}

\section{Methods}

A national cross-sectional online survey of Australian general practitioners was conducted in April and May 2020, with 572 respondents.

\section{Results}

The COVID-19 pandemic in Australia has resulted in major changes to general practice business models. Most practices have experienced increased workload and reduced income.

\section{Discussion}

Australian general practices have undertaken major innovation and realignment to respond to staff safety and patient care challenges during the COVID-19 pandemic. Increased administration, reduced billable time, managing staffing and pivoting to telehealth service provision have negatively affected practice viability. Major sources of information for general practice are primary care-specific, but many practices turn to colleagues for support and resources.
AT THE TIME OF WRITING this article in May 2020, the government, health service and community response to the COVID-19 pandemic in Australia had resulted in an extraordinarily low level of infection and death from SARS-CoV-2. At the end of May 2020, there had been just over 7000 diagnosed infections (282 per million population) and just over 100 deaths in Australia (four per million population), ${ }^{1}$ with approximately 1-3 new deaths per week. At the time, this compared favourably with pandemic death rates in some other developed countries, which were more than 100 times higher and rising. Unfortunately, the situation in Australia, and particularly in Victoria, changed markedly during the time this paper was being reviewed and revised in June-August 2020.

The successful population health outcomes correlate with enormous economic and social impacts in Australia. Major health service realignment has been required of general practices at the frontline of prevention and early intervention of the COVID-19 response. However, the nature of the general practice response, and any geographical variations, remains poorly described. Using a national online survey of general practitioners (GPs), this exploratory study investigates challenges, responses and effects of the COVID-19 pandemic in Australian general practice.

\section{Methods}

General Practice Supervisors Australia (GPSA), as the peak national body advocating for and supporting general practice supervisors, conducts an annual national survey of Australian general practice supervisors to inform policy and practice. This survey results in reports to GPs, government and regional training organisations (RTOs). ${ }^{2}$ GPSA focused the 2020 survey on the COVID-19 pandemic responses and challenges in Australian general practice.

The survey was developed by the GPSA research team and piloted with seven GPs. It gained ethics approval from Monash University (project ID number 19442). The survey consisted of 11 multi-part questions. These covered practice size, state/territory and locality, classified using the Modified Monash Model (MMM) categories; ${ }^{3}$ the practice response to COVID-19 and its pandemic plan; and the extent of challenges, responses and effects of COVID-19 in general practice. Open-ended responses allowed participants to describe what GPSA could do to assist them and note any other comments.

The survey was administered via GPSA's regular contact list $(\mathrm{n}=4891$ emails) using a SurveyMonkey link on 14 April 2020. Respondents could choose to enter a draw for an electronic gift voucher at the conclusion of the survey. Two email reminders were sent, and the survey closed after one month on 13 May 2020.

Analysis was conducted using SPSS Statistics 25 (SPSS Inc., Chicago). Percentage distributions were calculated for responses to each question, with responses for questions 4-9 weighted to the national distribution of GPs by 
state/territory and MMM4 to account for non-response bias. Multivariable logistic regression was used to test whether responses for each question were statistically significantly different by state/ territory or locality (controlling for practice size). A $P$ value of $<0.05$ was considered to denote statistical significance.

\section{Results}

In total, 572 participants completed the survey - a response rate of $12 \%$. Almost all respondents answered every closed-ended question; the lowest response number was for the question related to current number of GPs in the practice ( $n=551,96 \%)$. Median time to complete the survey was eight minutes.

\section{Practice characteristics and sample representativeness}

Survey responses are shown in Tables 1-4 and Figure 1 as percentage distributions. Table 1 shows size (number of GPs), state and locality of the respondents' main practices. Five per cent of respondents worked in a sole-GP practice, $41 \%$ in practices with 2-5 GPs, $34 \%$ in practices with 6-10 GPs and 20\% in practices with $\geq 11$ GPs. The state/territory distribution of respondents was similar to that of the GP population across Australia: $72 \%$ of respondents were from $\mathrm{New}$ South Wales (27\%), Victoria (25\%) or Queensland (20\%); 9\% each were from South Australia and Western Australia, $6 \%$ from Tasmania, and $2 \%$ each from the Northern Territory and the Australian Capital Territory. The main practice of $44 \%$ of respondents was in MMM1 (metropolitan areas), and 56\% were based in non-metropolitan areas (MMM2-7), which is overrepresented relative to the $28 \%$ of GPs working in Australia's MMM2-7 areas. ${ }^{4}$ This is adjusted for in the percentage distributions by weighting to national proportions of GPs by state/ territory and MMM. ${ }^{4}$

\section{Pandemic plans and other sources of information}

Overall, $57 \%$ of respondents stated that their practice had a pandemic plan in place before the COVID-19 pandemic. Half of those with a plan rated the applicability of this plan to the COVID-19 response as $\leq 50$ out of a possible score of zero to 100 (Table 1).

Free-text responses identified that existing plans were inadequate and not particularly relevant to the context of
COVID-19. Several respondents noted that their practice's pandemic plan was a re-purposed disaster, swine flu or epidemic influenza plan that had not been recently reviewed.

Respondents were also asked to select from a list of sources of information to

\section{Table 1. Practice characteristics}

\begin{tabular}{|c|c|}
\hline Characteristic & Percentage \\
\hline Practice state/territory $(n=572)$ & Unweighted \% \\
\hline New South Wales & 26.7 \\
\hline Victoria & 24.8 \\
\hline Queensland & 20.3 \\
\hline South Australia & 9.3 \\
\hline Western Australia & 9.4 \\
\hline Tasmania & 5.6 \\
\hline Northern Territory & 2.3 \\
\hline Australian Capital Territory & 1.6 \\
\hline Geographic area $(n=572)$ & Unweighted \% \\
\hline MMM1 (Metropolitan area) & 43.2 \\
\hline MMM2 (Regional centre) & 19.1 \\
\hline MMM3 (Large rural town) & 14.0 \\
\hline MMM4 (Medium rural town) & 10.7 \\
\hline MMM5 (Small rural town) & 10.1 \\
\hline MMM6\&7 (Remote/very remote community) & 3.0 \\
\hline Number of general practitioners in practice $(n=551)$ & Unweighted \% \\
\hline 1 & 4.5 \\
\hline $2-5$ & 40.8 \\
\hline $6-10$ & 34.1 \\
\hline$\geq 11$ & 20.5 \\
\hline Pandemic plan in place and applicability* $(n=572)$ & Weighted \% \\
\hline No & 42.1 \\
\hline Don't know & 1.0 \\
\hline Yes & 56.8 \\
\hline 0-25 applicable & 9.1 \\
\hline 26-50 applicable & 18.5 \\
\hline 51-75 applicable & 17.1 \\
\hline 76-100 applicable & 10.8 \\
\hline Data unavailable & 1.2 \\
\hline
\end{tabular}


indicate which they have used to inform their practice's response to COVID-19. More than three-quarters of respondents indicated that their sources of information include The Royal Australian College of General Practitioners (RACGP; 86\%), state government (79\%), Australian Federal Government (77\%) and local primary health networks (77\%). The practice's pandemic plan was selected by $33 \%$ of respondents (Table 2).

\section{Challenges, responses and effects of COVID-19 on general practice}

Results regarding the potential challenges encountered by general practice during the COVID-19 pandemic are shown in Table 3. More than two-thirds of respondents reported that the following challenges had had a high or medium impact on their practices: managing the safety of staff ( $86 \%)$, disinfecting equipment and surfaces (86\%), lack of full personal protective equipment (80\%), managing patients $(78 \%)$, community self-isolating as instructed and not seeking care from their GP as they normally would (76\%), lack of face masks
(73\%), telehealth reimbursement (74\%) and consistency of information (71\%).

Other challenges ranked as having a high/medium impact by more than half of respondents were: managing patient demands (65\%), increased overall workload $(61 \%)$, getting information when needed (59\%), timely access to patient COVID-19 test results (53\%) and lack of testing equipment (52\%). Challenges with a lower impact were those associated with medical student and registrar placements, patient transfers, technology infrastructure, lack of staff, staff working from home or self-isolating, and decreased workload (Table 3).

The most common responses (high/ medium use) to the pandemic were: telehealth after the Medicare Benefits Schedule (MBS) items became available (97\%), COVID-19-specific signage (94\%), new cleaning protocols (93\%), segregating unwell patients with COVID-19 and/or influenza-like symptoms (91\%), reception triage (94\%), nurse triage (70\%), asking colleagues for information and sharing resources $(77 \%)$, additional preventive services for elderly clients (71\%),

\section{Table 2. Sources of information for practice response to the COVID-19 pandemic $(n=572)$}

Sources of information Weighted \%

The Royal Australian College of General Practitioners (RACGP)

State government, including state departments of health

Local Primary Health Network (PHN)

Australian Federal Government, including the Australian Department of Health

General Practice Supervisors Australia (GPSA)

Australian Medical Association (AMA) 39.9

World Health Organization (WHO) 33.4

My general practice's pandemic plan 32.7

My general practice's Regional Training Organisation (RTO)

Practice Managers Network (Facebook)

23.6

GPs Down Under (GPDU)

20.5

Australian College of Rural and Remote Medicine (ACRRM)

Local/state Aboriginal Community Controlled Health Organisation on-referral for testing $(71 \%)$, daily staff (de)briefings (71\%), and registrars engaged in the response (67\%; Table 4).

Respondents indicated that their practices had been substantially affected by the pandemic in terms of: decreases in bookings ( $73 \%$ of respondents) and practice income $(77 \%)$; and increases in practice costs (81\%), phone calls (93\%), non-clinical staff time $(76 \%)$ and non-billable time and activity (74\%; Figure 1).

\section{Differences by state or locality}

Although infections and deaths from COVID-19 in Australia have varied enormously across Australia, multivariable logistic regression analysis did not reveal recurring differences by state/territory, controlling for locality and practice size. However, some differences did emerge by locality.

When compared with practices in metropolitan areas, practices in non-metropolitan areas were more likely to source information for their practice's pandemic response from their RTO and the Australian College of Rural and Remote Medicine, and less likely to source information from the RACGP and their local primary health network.

Respondents in metropolitan areas reported some challenges more often than doctors in non-metropolitan areas: receiving information when needed, and consistency of information; lack of testing equipment, personal protective equipment and face masks; disinfecting equipment and surfaces; and managing staff safety. Respondents in MMM5-7 (small rural towns; remote/very remote communities) were more likely to select patient transfers as a challenge, compared with respondents in MMM1 (metropolitan areas).

In terms of the COVID-19 response, metropolitan-based respondents were more likely than non-metropolitan respondents to ask colleagues for information/share resources, employ on-referral for testing and roster on more staff; and less likely to employ ambulatory clinics (eg to nursing homes), use nurse triage and engage medical students in the practice's pandemic response. Effects of the COVID-19 pandemic were greater in metropolitan practices in 
terms of increased activity and costs, and decreased bookings.

\section{Discussion}

This is one of the first national studies profiling the general practice response to COVID-19 and identifying the unique challenges and opportunities that have emerged. General practice pandemic plans were either unavailable or not relevant in the COVID-19 context. This is concerning and will need to be addressed in future policy and practice, taking into account accreditation requirements and associated resources and costs for implementation. Major COVID-19 information sources relied on in general practice include the colleges, government and primary health networks. The degree of consistent and real-time messaging between these groups may require specific attention to make it easy for practices to navigate and apply practical information. An area-level response, in which practices in the same community share information, may also be useful, given

Table 3. Extent to which practices were impacted by each of the following challenges during the current pandemic $(n=572)$

\begin{tabular}{|c|c|c|c|c|c|c|}
\hline & $\begin{array}{r}\text { High/medium } \\
\text { impact }\end{array}$ & $\begin{array}{r}\text { High } \\
\text { impact }\end{array}$ & $\begin{array}{r}\text { Medium } \\
\text { impact }\end{array}$ & $\begin{array}{r}\text { Low } \\
\text { impact }\end{array}$ & $\begin{array}{r}\text { No } \\
\text { impact }\end{array}$ & $\begin{array}{r}\text { Not } \\
\text { applicable }\end{array}$ \\
\hline Challenge & \multicolumn{6}{|c|}{ Percentage distribution (weighted) } \\
\hline Disinfecting equipment and surfaces & 85.7 & 46.2 & 39.4 & 11.7 & 2.4 & 0.2 \\
\hline Lack of full personal protective equipment & 80.4 & 52.4 & 28.0 & 13.8 & 5.2 & 0.5 \\
\hline $\begin{array}{l}\text { Community self-isolating as instructed and not } \\
\text { seeking care from their general practitioner as they } \\
\text { normally would }\end{array}$ & 75.9 & 37.1 & 38.8 & 19.1 & 4.0 & 1.0 \\
\hline Telehealth reimbursement & 74.1 & 38.8 & 35.3 & 18.2 & 7.2 & 0.5 \\
\hline Increased overall workload & 61.3 & 27.7 & 33.5 & 22.2 & 12.9 & 3.7 \\
\hline Getting information when needed & 59.1 & 13.5 & 45.6 & 29.4 & 10.8 & 0.7 \\
\hline Timely access to patient COVID-19 test results & 53.3 & 17.8 & 35.5 & 34.4 & 10.5 & 1.7 \\
\hline Lack of testing equipment & 51.8 & 24.7 & 27.1 & 28.0 & 12.4 & 7.7 \\
\hline Lack of patient consults for registrars & 46.5 & 17.7 & 28.8 & 24.7 & 14.7 & 14.2 \\
\hline Lack of technology infrastructure & 44.7 & 11.9 & 32.8 & 35.1 & 16.6 & 3.7 \\
\hline Meeting educational/supervision needs of registrars & 40.2 & 10.7 & 29.5 & 31.5 & 14.3 & 14.0 \\
\hline Patient transfer issues & 26.0 & 9.8 & 16.3 & 38.1 & 23.8 & 12.1 \\
\hline Lack of other practice staff & 23.7 & 6.5 & 17.3 & 36.1 & 33.5 & 6.6 \\
\hline Lack of medical staff & 21.2 & 5.2 & 15.9 & 38.8 & 34.6 & 5.4 \\
\hline $\begin{array}{l}\text { The practice has stopped taking medical students } \\
\text { (practice choice) }\end{array}$ & 9.3 & 4.2 & 5.1 & 7.5 & 16.6 & 66.6 \\
\hline
\end{tabular}


that $77 \%$ of respondents asked colleagues for information/shared resources on responding to the COVID-19 crisis.

Major concerns about staff and patient safety were apparent, with anxieties potentially exacerbated by lack of resources such as personal protective equipment and delays in receiving timely test results. As first-contact practitioners, GPs are most susceptible to pandemics. They rely heavily on community compliance with self-isolation and manage potentially infectious patients, making for a challenging working environment. They must encourage patients to get the care they need while mitigating the risk involved in seeing an undifferentiated COVID-19 caseload. Practices were highly adaptable. Many used regular staff meetings and coped with increased workloads associated with implementing standardised cleaning and clinical regimens, and dealing with a large increase in community anxiety, including phone calls to the practice.
With respect to practice viability, new MBS items for telehealth have been widely employed, but the shift to these using bulk billing only may have reduced practice revenue. Most respondents also reported decreased bookings and practice income, coupled with increased practice costs, likely related to increased administrative burden of non-clinical staff, non-billable activity and paying for resources.

The results show a consistent pandemic response by general practices across

Table 4. Extent to which practices used the following responses during the crisis $(n=572)$

High/medium

use

Response

Telehealth (video/telephone) after MBS items became available

COVID-19-specific signage

Reception triage

Introduction of new cleaning protocols

Segregation of unwell patients with COVID-19 and/or influenza-like symptoms

Asking colleagues for information/sharing resources

On-referral for testing

Additional preventative services for elderly clients

Daily staff briefings/de-briefing

Nurse triage

Registrars engaged in practice's pandemic response

Working longer hours

Telehealth (video/telephone) before MBS items became available

Pop-up, fever, URTI clinics

Reduced clinical staff hours

Registrar triage

Ambulatory clinics (eg to nursing homes)

Roster on more staff

Drive-through testing

Not taking on a registrar in Term 2, 2020

Medical students engaged in practice's pandemic response

Discontinuing employ of our existing registrar(s) in the current term

MBS, Medicare Benefits Schedule; NA, not applicable; URTI, upper respiratory tract infection

High Medium Low

use use use

NA

Percentage distribution (weighted)

\begin{tabular}{lllll}
96.9 & 77.7 & 19.2 & 2.4 & 0.7 \\
\hline 94.0 & 80.0 & 14.0 & 4.4 & 1.6 \\
94.0 & 75.3 & 18.7 & 5.6 & 0.4 \\
\hline 93.2 & 68.2 & 25.0 & 5.8 & 1.0 \\
90.9 & 72.1 & 18.8 & 7.3 & 1.7
\end{tabular}

77.100 .0

\begin{tabular}{lllll}
77.1 & 36.0 & 41.2 & 20.1 & 2.8 \\
\hline
\end{tabular}

$\begin{array}{lllll}71.5 & 37.1 & 34.3 & 22.8 & 5.8\end{array}$

$\begin{array}{lllll}71.1 & 38.4 & 32.7 & 22.8 & 6.1\end{array}$

$\begin{array}{lllll}71.0 & 30.9 & 40.0 & 24.5 & 4.5\end{array}$

$\begin{array}{lllll}69.9 & 40.5 & 29.4 & 21.4 & 8.8\end{array}$

$\begin{array}{lllll}67.3 & 33.6 & 33.7 & 15.2 & 17.5\end{array}$

$\begin{array}{lllll}60.7 & 29.7 & 31.1 & 26.5 & 12.7 \\ 54.6 & 31.5 & 23.1 & 30.5 & 14.9\end{array}$

$\begin{array}{lllll}54.6 & 31.5 & 23.1 & 30.5 & 14.9 \\ 37.1 & 19.4 & 177 & 23.6 & 39.3\end{array}$

$\begin{array}{lllll}29.9 & 8.7 & 21.2 & 38.5 & 31.6\end{array}$

\begin{tabular}{lllll}
29.9 & 8.7 & 21.2 & 38.5 & 31.6 \\
\hline
\end{tabular}

\begin{tabular}{lllll}
26.9 & 10.0 & 17.0 & 36.4 & 36.7 \\
\hline
\end{tabular}

$\begin{array}{lllll}26.2 & 9.3 & 17.0 & 35.5 & 38.3\end{array}$

$\begin{array}{lllll}24.1 & 8.6 & 15.5 & 28.1 & 47.8\end{array}$

\begin{tabular}{lllll}
24.1 & 8.6 & 15.5 & 28.1 & 47.8 \\
\hline 18.0 & 8.0 & 10.0 & 20.5 & 61.5
\end{tabular}

\begin{tabular}{lllll}
8.0 & 8.0 & 10.0 & 20.5 & 61.5 \\
\hline 0.6 & 5.4 & 5.2 & 15.0 & 74.3
\end{tabular}

\begin{tabular}{rrrrr}
7.7 & 2.6 & 5.1 & 15.0 & 74.3 \\
\hline 4.4 & 2.1 & 2.3 & 14.3 & 79.2 \\
\hline
\end{tabular}


Australia, with some differences according to rurality. The significant financial effects related to revenue were most felt in metropolitan areas.

\section{Limitations}

The survey response rate was low (12\% of emails sent). However, surveys of medical practitioners generally have relatively low response rates. The well-publicised Medicine in Australia: Balancing Employment and Life (MABEL) survey had a baseline response rate of $18 \%$ for GPs, ${ }^{5}$ and other surveys of medical practitioners have reported response rates as low as $9 \%{ }^{6}$ Response rates may have been affected by the COVID-19 environment, which added time pressures on GPs because of changing practices and fielding queries from primary health networks, health departments, other agencies and patients. Rural and remote practices may have also been managing the consequences of other recent crises such as drought and bushfires.

The study surveyed practices that provide formal supervision of general practice registrars, which may not be representative of all general practices. Respondents in non-metropolitan practices were overrepresented. Survey results were weighted to account for this; however, if GPs in metropolitan areas with particularly high stressors were less likely to answer the survey, it is possible that the analysis underestimates the level of challenges, responses and effects of COVID-19 in Australian general practice. The survey is a snapshot of perceptions of survey respondents in late April and early May 2020, and may not reflect the changing situation as the pandemic progresses. This exploratory study may inform future pandemic preparedness and related research.

\section{Conclusion}

This exploratory study suggests a consistent and relatively common level of experience across Australian general practices, highlighting that the pandemic has required a major realignment of business to manage staffing, resources and different cohorts of patients to achieve safety and quality. Putting patients and the community first, many general practices may have experienced business viability challenges. Ongoing pandemic management, and organisation and support of general practice, may demand that the role of general practice in pandemic responsiveness is more clearly articulated and any business stressors are managed.

\section{Authors}

Rebecca Kippen BBus, PhD, Associate Professor of Demography, School of Rural Health, Monash University, Vic. rebecca.kippen@monash.edu

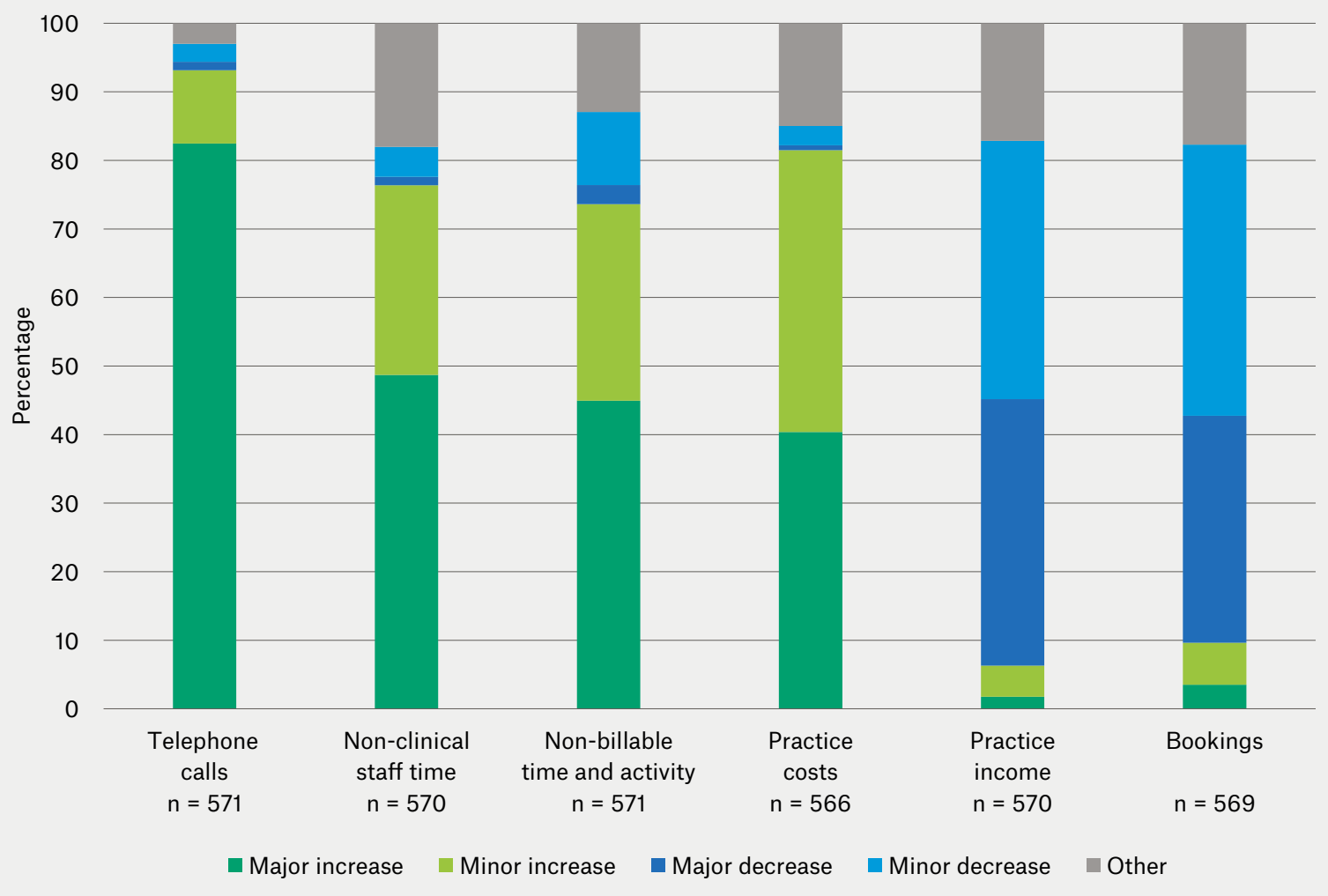

Figure 1. Extent to which practices have been affected by the pandemic response so far 
Belinda O'Sullivan BPhysio (Hons), MPH (Hons), GradDipAppEpi, PhD, Research Fellow, Rural Clinical School, University of Queensland, Qld

Helen Hickson BSocWork, PhD, Research Fellow, General Practice Supervisors Australia, Vic

Michael Leach BPharm, GradCertSc(App Stat), MBiostat, CHIA, PhD, Senior Lecturer, School of Rural Health, Monash University, Vic

Glen Wallace MBA, GradCertMgmt, MAICD, Chief Executive Officer, General Practice Supervisors Australia, Vic

Competing interests: RK reports education research grants related to GP education from The Royal

Australian College of General Practitioners, outside the submitted work. HH reports she was employed by General Practice Supervisors Australia (GPSA) but the views expressed are her own and are not influenced by GPSA.

Funding: Authors received funding from the Australian Federal Government under the Australian General Practice Training Program through General Practice Supervisors Australia to conduct this research.
Provenance and peer review: Not commissioned, externally peer reviewed.

\section{Acknowledgements}

The researchers thank the survey respondents for their time and expertise. The researchers also thank the editor and five anonymous reviewers for their careful reading of the article and their constructive comments and suggestions, which have significantly improved this manuscript.

\section{References}

1. Department of Health. Coronavirus (COVID-19) health alert. Canberra, ACT: DoH, 2020. www.health.gov.au/news/health-alerts/ novel-coronavirus-2019-ncov-health-alert [Accessed 2 September 2020].

2. General Practice Supervisors Australia. National GP supervisor survey: National results. Bendigo, Vic: GPSA, 2017.
3. Department of Health. Modified Monash model. Canberra, ACT: DoH, 2020 www.health. gov.au/health-workforce/health-workforceclassifications/modified-monash-model [Accessed 2 September 2020].

4. Department of Health. Health workforce data. Canberra, ACT: DoH, 2020. Available at https:// hwd.health.gov.au [Accessed 2 September 2020].

5. Joyce C, Scott A, Jeon SH, et al. The 'Medicine in Australia: Balancing Employment and Life (MABEL)' longitudinal survey - Protocol and baseline data for a prospective cohort study of Australian doctors' workforce participation. BMC Health Serv Res 2010;10:50. doi: 10.1186/14726963-10-50.

6. Aitken C, Power R, Dwyer R. A very low response rate in an on-line survey of medical practitioners. Aust N Z J Public Health 2008;32(3):288-89. doi: 10.1111/j.1753-6405.2008.00232.x. 